\title{
Are circulating microRNAs suitable for the early detection of malignant mesothelioma? Results from a nested case- control study
}

\author{
Daniel Gilbert Weber ${ }^{*}$, Alexander Brik, Swaantje Casjens, Katarzyna Burek, Martin Lehnert, Beate Pesch, \\ Dirk Taeger, Thomas Brüning, Georg Johnen and the MoMar study group
}

\begin{abstract}
Objective: Malignant mesothelioma is an aggressive cancer of the serous membranes. For the detection of the tumor at early stages non- or minimally-invasive biomarkers are needed. The circulating biomarkers miR-132-3p, miR126-3p, and miR-103a-3p were analyzed in a nested case-control study using plasma samples from 17 prediagnostic mesothelioma cases and 34 matched asbestos-exposed controls without a malignant disease.

Results: Using prediagnostic plasma samples collected in median 8.9 months prior the clinical diagnosis miR-132-3p, miR-126-3p, and miR-103a-3p revealed $0 \%$ sensitivity on a defined specificity of $98 \%$. Thus, the analyzed miRNAs failed to detect the cancer in prediagnostic samples, showing that they are not feasible for the early detection of malignant mesothelioma. However, the miRNAs might still serve as possible markers for prognosis and response to therapy, but this needs to be analyzed in appropriate studies.
\end{abstract}

Keywords: Biomarker, Blood, Cancer, Liquid biopsies, Mesothelioma, MicroRNA, Marker, Negative results, Null results, Prediagnostic

\section{Introduction}

Malignant mesothelioma is an asbestos-related malignancy with increasing incidence worldwide [1]. Based on the continued use of asbestos and a long latency period of up to 50 years mesothelioma remains a global health problem. Commonly, symptoms occur at late stages of the disease and median survival after diagnosis is between nine and 13 months, depending on treatment [2]. A diagnosis of mesothelioma at early stages, when the tumor is still small and has not spread, might be a promising opportunity to improve therapy options. Current therapies are improved in rather small steps [3] and promising approaches like immunotherapy are being investigated [4]. Notably, Jones et al. presented a case

*Correspondence: weber@ipa-dguv.de

Institute for Prevention and Occupational Medicine of the German

Social Accident Insurance, Institute of the Ruhr University Bochum (IPA),

Buerkle-de-la-Camp-Platz 1, 44789 Bochum, Germany with an exceptional and sustained response to immune checkpoint inhibition [5]. In order to finally reduce mortality there is a need to identify and validate appropriate biomarkers for the early detection of cancer, particularly for mesothelioma [6].

To detect cancer at early stages, non- and minimallyinvasive methods like liquid biopsies are preferable. Circulating miRNAs are well-known biomarkers of several diseases including cancer and might be feasible for early detection [7], e.g., liver cancer was detected prior to a common clinical diagnosis using circulating miRNAs [8, 9]. For the detection of malignant mesothelioma miR-16 [10], miR-17 [10], miR-30e-3p [11], miR-103a-3p [12], miR-126 [13], miR-625-3p [14], miR-132-3p [15], and miR-486 [10] were introduced as blood-based biomarkers. However, the miRNAs in those studies were analyzed using cross-sectional designs, commonly resulted in study groups with higher numbers of cancer patients 
at late stages. Thus, the biomarkers do not allow to draw any conclusion regarding their performance for the detection of mesothelioma at early stages [16]. Most of the introduced biomarker candidates are not validated in samples collected prior to a diagnosis of malignant mesothelioma. Hence, longitudinal studies, which facilitate repeated sampling of an at-risk population, are important for the evaluation of the biomarker performance for the early detection of the cancer.

The aim of this study was to analyze circulating miRNAs in a case-control study nested into a prospective cohort to assess the biomarker performance for the detection of malignant mesothelioma in prediagnostic plasma samples.

\section{Main text Methods}

The MoMar (Molecular Markers) cohort consists of 2769 German workers formerly exposed to asbestos with a confirmed asbestos-related occupational disease (asbestosis and/or other (nonmalignant) pleural diseases caused by asbestos). The participants were recruited from November 2008 to February 2018 at 26 medical centers in Germany. Voluntary blood donation and a questionnaire were offered every year [17]. Using a nested casecontrol design, 17 male mesothelioma patients including ten epithelioid (58.8\%), two biphasic (11.8\%), and three sarcomatoid (17.6\%) mesotheliomas were investigated. In two cases $(11.8 \%)$ the histological subtype of the tumor remained unknown. The median time between blood collection and date of diagnosis was 8.9 months. To each case two cancer-free controls were matched. Thus, the control group comprised 34 men of the MoMar cohort. Criteria for matching were gender, age, smoking status, and date of blood collection. Characteristics of the study groups are presented in Additional file 1 and detailed characteristics of the subjects in Additional file 2.

Peripheral blood was collected in $9.0 \mathrm{ml} \mathrm{S}$-Monovette EDTA gel tubes (Sarstedt, Nümbrecht, Germany). Within $30 \mathrm{~min}$ after blood collection samples were centrifuged at $2000 \times g$ for $10 \mathrm{~min}$ at room temperature. After centrifugation plasma was separated from the cellular fraction and both matrices were immediately frozen and temporarily stored in the collaborating study centers. Samples were regularly picked up, transported to the central laboratory, aliquoted using an automated liquid handling robot (Tecan Group Ltd., Männedorf, Switzerland), and stored at $-80{ }^{\circ} \mathrm{C}$ until use. For the determination of miR132-3p and miR-126-3p, RNA from $0.5 \mathrm{ml}$ plasma was isolated using the miRVana PARIS kit (Thermo Fisher Scientific, Darmstadt, Germany) according to the manufacturer's instructions, modified by adding $5 \mu \mathrm{l}$ Carrier RNA MS2 (Roche, Mannheim, Germany). For the determination of miR-103a-3p, RNA from $0.5 \mathrm{ml}$ cellular fraction was isolated using the RiboPure-Blood Kit according to the Alternate protocol: Isolation of Small RNAs (Thermo Fisher Scientific). Individual miRNAs were analyzed using commercial TaqMan microRNA assays (Life Technologies) for miR-103a-3p (ID 000439), miR-125a (ID 000448), miR-132-3p (ID 000457), miR146b-5p (ID 001097), miR-126-3p (ID 002228), and U6 snRNA (ID 001973) as described previously [15]. Quantitative miRNA expression data were acquired using the ABI SDS software (Thermo Fisher Scientific) and presented in Additional file 2. Estimation of the cycle threshold $(\mathrm{Ct})$ was performed as described elsewhere [12, 15]. Normalization was performed with the $2^{-\Delta \mathrm{Ct}}$ method [18] using miR-146b-5p, U6 snRNA, and miR-125a, as references for miR-132-3p, miR-126-3p, and miR$103 a-3 p$, respectively.

Box plots with median and inter-quartile range (IQR) were used to depict the distribution of single biomarkers and their combination. Whiskers represent minimum and maximum values. Mann-Whitney $U$ tests or Kruskal-Wallis tests were applied to examine group differences and $\mathrm{p}$-values $<0.05$ were considered as statistically significant. Receiver operating characteristic (ROC) curves were used to quantify classification performance of the biomarkers. The accuracy of the diagnostic tests was depicted by the area under curve (AUC) and its 95\% confidence interval (CI). The ROC curves of the biomarker combination were calculated with each miRNA as independent variable in a multiple logistic regression model. Sensitivities and specificities were determined using a defined specificity of $98 \%$ or maximum Youden's Index (YI). All statistical analyses were performed using SAS/STAT and SAS/IML software, version 9.4 (SAS Institute Inc., Cary, USA). Graphs were generated using GraphPad Prism (GraphPad Software, La Jolla, USA).

\section{Results}

The two miRNAs miR-132-3p and miR-126-3p were determined in prediagnostic plasma samples. The median level of miR-132-3p was 0.015 (IQR 0.012-0.020) for mesothelioma cases and 0.016 (IQR 0.012-0.024) in the control group (Fig. 1a). The median level of miR126-3p was 2925 (IQR 878-4012) in the mesothelioma group and 1413 (IQR 600-3170) for cancer-free controls (Fig. 1b). In two control subjects miR-126-3p could not be determined. The miRNA miR-103a-3p was measured in the cellular fraction of prediagnostic blood samples. The median level of miR-103a-3p was 282 (IQR 221-523) in mesothelioma cases and 471 (IQR 228-707) in the control group (Fig. 1c). In one control subject miR-103a-3p could not be determined. Group differences were not statistically significant for any tested 

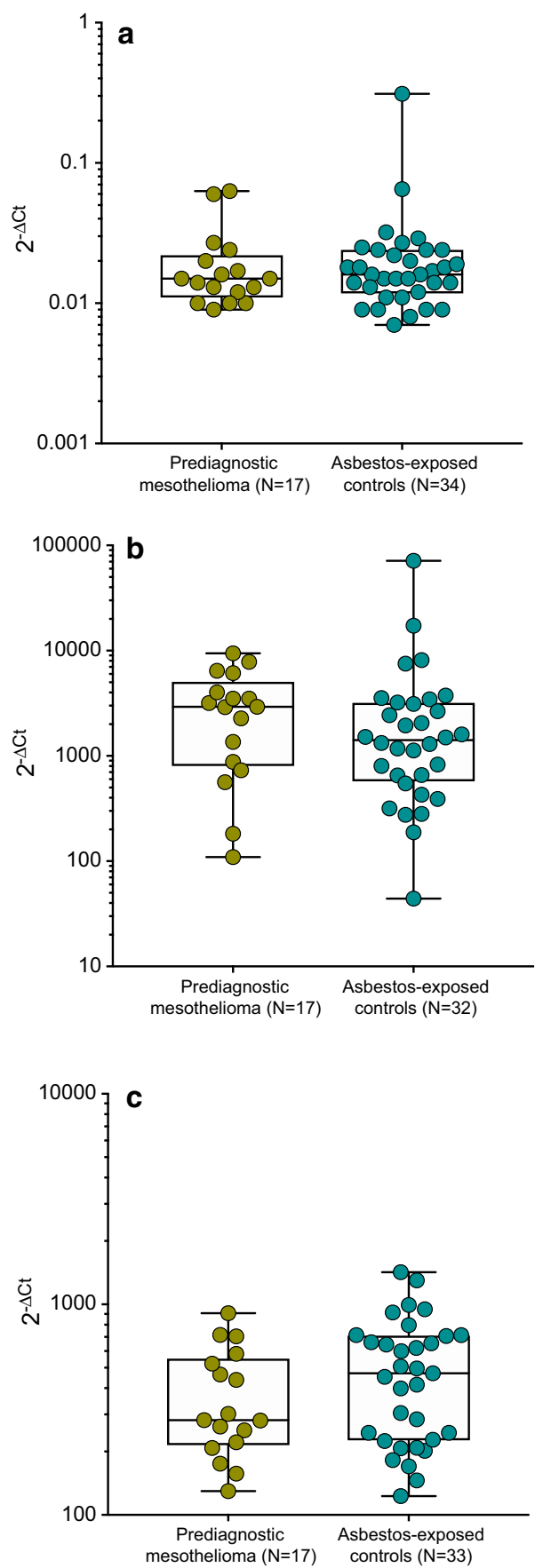

Fig. 1 Distribution of miR-132-3p (a), miR-126-3p (b), and miR-103a-3p (c) in prediagnostic mesothelioma cases and asbestos-exposed controls. Mann-Whitney $\mathrm{U}$ tests were performed to examine group differences. Horizontal bars represent median and interquartile range

miRNA. Additionally, prediagnostic mesothelioma cases were divided in three subgroups according to the time between blood collection and diagnosis $[<6$ months $(\mathrm{N}=5), 6-12$ months $(\mathrm{N}=7)$, and $\geq 12$ months $(\mathrm{N}=5)]$.

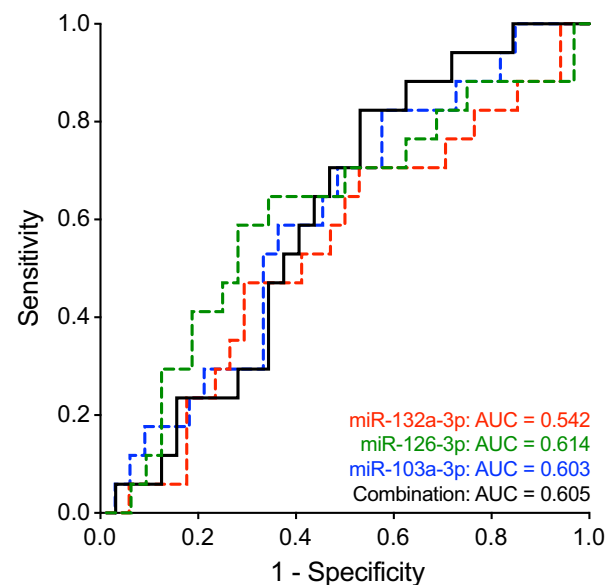

Fig. 2 Receiver operating characteristic (ROC) curves of miR-132-3p, miR-126-3p, miR-103a-3p, and the combination of the three miRNAs

Table 1 Sensitivities and specificities of miR-132a-3p, miR126-3p, miR-103a-3p, and the combination of the three miRNAs

\begin{tabular}{lcl}
\hline Biomarker & Sensitivity (\%) & Specificity (\%) \\
\hline miR-132-3p & & \\
Defined specificity & 0 & 98 \\
Maximum YI & 71 & 47 \\
miR-126-3p & & \\
Defined specificity & 0 & 98 \\
Maximum YI & 59 & 72 \\
miR-103a-3p & & \\
Defined specificity & 0 & 98 \\
Maximum Yl & 82 & 42 \\
Combination of the three miRNAs & \\
Defined specificity & 0 & 98 \\
Maximum Yl & 82 & 47
\end{tabular}

No statistically significant differences between the groups could be observed for any analyzed miRNA (Additional file 3).

The ROC analyses showed an AUC of 0.542 (95\% CI 0.370-0.713) for miR-132a-3p, 0.614 (95\% CI 0.439 0.789 ) for miR-126-3p, and 0.603 (95\% CI $0.440-0.765$ ) for miR-103a-3p (Fig. 2). Using a defined specificity of $98 \%$, a sensitivity of $0 \%$ was revealed for each miRNA. Using maximum YI resulted in $71 \%, 59 \%$, and $82 \%$ sensitivity and $47 \%, 72 \%$, and $42 \%$ specificity for miR-132-3p, miR-126-3p, and miR-103a-3p, respectively (Table 1 ). The combination of all three miRNAs revealed an AUC of 0.605 (95\% CI 0.445-0.765). The combined ROC curve is displayed in Fig. 2. Using a defined specificity of $98 \%$ resulted in $0 \%$ sensitivity and using maximum YI resulted 
in $82 \%$ sensitivity and $47 \%$ specificity for the biomarker combination (Table 1).

\section{Discussion}

For cancer detection in prediagnostic samples, high specificities of the biomarkers are needed to receive a preferable low number of false-positive tests in order to avoid psychological distress and unnecessary invasive examinations for the patients [16]. Using a defined specificity of $98 \%$ none of the mesothelioma cases were detected by miR-132-3p and miR-126-3p in plasma, as well as miR103a-3p in the cellular fraction of blood. Similarly, using maximum YI resulted in low sensitivities and specificities. Also, the combination of the three biomarkers did not improve the marker performance. Thus, the analyzed candidate biomarkers failed to detect malignant mesothelioma in prediagnostic blood samples, showing that they are not feasible for the early detection of this cancer. Originally, for miR-132-3p, miR-126-3p, and miR103a-3p promising sensitivities of $86 \%, 61 \%$, and $83 \%$, and specificities of $73 \%, 74 \%$, and $71 \%$, respectively, were revealed for the discrimination of mesothelioma patients and asbestos-exposed controls [12, 13, 15]. However, compared to the results obtained with already manifest tumors, the performance of the analyzed miRNAs dropped substantial using prediagnostic samples.

Globally, miRNAs are down-regulated in cancer patients [19] and the same trend could be observed for miR-132-3p, miR-126-3p, and miR-103a-3p in manifest mesothelioma $[12,13,15]$. However, the down-regulation of circulating miRNAs is not a result of a downregulation of these miRNAs within the tumor [20]. The tumor growth might rather negatively affect the miRNA expression in other body cells releasing the miRNAs. Thus, the down-regulation of circulating miRNAs might be a non-specific response to the presence of neoplastic growth [20]. It remains plausible that the tumor needs to have a sufficient size for a distinct effect on the miRNA expression in other cells. The analyzed blood samples in this study were taken from prediagnostic mesothelioma patients on average 8.9 months prior to the clinical diagnosis based on symptoms or a health impairment. Thus, the tumor size might be comparatively small at the time of blood collection. It is comprehensible that the expression of the circulating miRNAs is not yet down-regulated in prediagnostic cancer samples in contrast to the initial identification studies using samples from manifest mesothelioma cases $[12,13,15]$.

However, circulating miRNAs are described as generally suitable to predict prognosis and response to therapy [21] and this might be also true for miR-132-3p, miR-126-3p, and miR-103a-3p. Thus, appropriate studies have to be carried out to assess the performance of the miRNAs for these applications.

For the detection of cancer at early stages, the use of circulating biomarkers in prediagnostic samples remains to be a meaningful approach, because biomarkers are economical, easy to apply, and might be implemented in clinical routine without much effort. Blyuss et al. demonstrated that the combination of CA125, HE4, and glycodelin detected ovarian cancer in blood samples up to 1 year before the clinical diagnosis with a sensitivity and specificity $>90 \%$ [22]. For malignant mesothelioma, Johnen et al. showed that the combination of mesothelin and calretinin detected the tumor using prediagnostic plasma samples up to 15 months prior the clinical diagnosis with a sensitivity of $46 \%$ at a defined specificity of $98 \%$ [17]. In contrast to the analyzed circulating miRNAs, the above-mentioned proteins might be released directly from the tumor, resulting in a sustainable detection even at early stages of the disease. The combination of several biomarkers within a panel improves the diagnostic performance [17, 22-25]. Thus, it is still important to identify and validate more biomarkers of different molecular classes, i.e. proteins as well as miRNAs, circular RNAs, long non-coding RNAs, and DNA-Methylation, for the detection of mesothelioma at early stages.

\section{Limitations}

Generally, in studies with a longitudinal design the number of incident cases of mesothelioma during follow-up is low. At the time point of the realized analyses only 17 samples from prediagnostic cancer cases that were diagnosed during a period of about 9 years were included.

Only three miRNAs were analyzed in this study, although miR-16, miR-17, and miR-486 [10], miR-30e-3p [11], and miR-625-3p [14] were also suggested as bloodbased biomarkers for mesothelioma. However, miR-16, miR-17, and miR-486 seem to be affected by hemolysis [26-28] making them not appropriate as blood-based biomarker in clinical routine. MiR-30e-3p were described as candidate biomarkers using extracellular vesicles. Unfortunately, the isolation of vesicles from plasma samples was not part of this study. MiR-625-3p was not analyzed in this study, because the initially described biomarker performance could not be verified in an independent study [15].

\section{Additional files}

Additional file 1. Characteristics of prediagnostic mesothelioma cases and asbestos-exposed controls in the nested case-control study.

Additional file 2. Detailed characteristics of the study subjects and quantitative miRNA expression data. 
Additional file 3. Distribution of miR-132-3p (A), miR-126-3p (B), and miR-103a-3p (C) in prediagnostic mesothelioma cases, categorized by time between blood collection and diagnosis ( $<6$ months, $6-12$ months, and $\geq 12$ months). Kruskal-Wallis tests were performed to examine group differences. Horizontal bars represent median and interquartile range.

\section{Abbreviations}

AUC: area under curve; Cl: confidence interval; Ct: cycle threshold; IQR: interquartile range; miRNA: microRNA; ROC: receiver operating characteristics; $\mathrm{Yl}$ : Youden's Index.

\section{Authors' contributions}

DGW conceived of this study, participated in its design and coordination, and drafted the manuscript. AB performed the experiments and helped to draft the manuscript. SC and KB analyzed the data and helped to draft the manuscript. ML, BP, DT, and TB participated in study design and coordination. GJ participated in study design, coordination, and helped to draft the manuscript. All authors read and approved the final manuscript.

\section{Acknowledgements}

We are grateful to our clinical colleagues for their support in obtaining human blood samples. We thank the trustees Ulrike Nissen and Sandra Ziob for their excellent cooperation.

The MoMar Study Group members (besides the authors) are Bettina Dumont, Jan Gleichenhagen, Olaf Hagemeyer, Heike Heimann, Evelyn Heinze, Monika Kobek, Claudia Lechtenfeld, Swetlana Meier, Carmen Meinig, Simone Naumann, Simone Putzke, Irina Raiko, Hans-Peter Rihs, Peter Rozynek, Sandra Schonefeld, Katja Szafranski, Katharina Wichert, Thorsten Wiethege, and Sandra Zilch-Schöneweis.

We acknowledge support by the DFG Open Access Publication Funds of the Ruhr University Bochum.

\section{Competing interests}

The authors declare that they have no competing interests.

\section{Availability of data}

All data generated or analyzed during this study are included in this published article and its additional files.

\section{Consent of publication}

Not applicable.

\section{Ethics approval and consent to participate}

The study was designed according to the rules guarding patient privacy and with the approval from the ethics committee of the Ruhr University Bochum (Reference Number 3217-08). All participants provided written informed consent

\section{Funding}

Not applicable.

\section{Publisher's Note}

Springer Nature remains neutral with regard to jurisdictional claims in published maps and institutional affiliations.

Received: 10 January 2019 Accepted: 6 February 2019

Published online: 11 February 2019

\section{References}

1. Robinson BM. Malignant pleural mesothelioma: an epidemiological perspective. Ann Cardiothorac Surg. 2012;1(4):491-6.

2. Hodgson JT, McElvenny DM, Darnton AJ, Price MJ, Peto J. The expected burden of mesothelioma mortality in Great Britain from 2002 to 2050. Br J Cancer. 2005:92(3):587-93.
3. Zalcman G, Mazieres J, Margery J, Greillier L, Audigier-Valette C, MoroSibilot D, Molinier O, Corre R, Monnet I, Gounant V, et al. Bevacizumab for newly diagnosed pleural mesothelioma in the Mesothelioma Avastin Cisplatin Pemetrexed Study (MAPS): a randomised, controlled, open-label, phase 3 trial. Lancet. 2016;387(10026):1405-14.

4. Brosseau S, Dhalluin X, Zalcman G, Scherpereel A. Immunotherapy in relapsed mesothelioma. Immunotherapy. 2018;10(2):77-80.

5. Jones RG, Karthik F, Dugar A, Kanagarajan K, Desai K, Bhandari M. Nivolumab immunotherapy in malignant mesothelioma: a case report highlighting a new opportunity for exceptional outcomes. Am J Case Rep. 2018;19:783-9.

6. Ahmadzada T, Reid G, Kao S. Biomarkers in malignant pleural mesothelioma: current status and future directions. Journal of thoracic disease. 2018;10(Suppl 9):S1003-7.

7. Wang H, Peng R, Wang J, Qin Z, Xue L. Circulating microRNAs as potential cancer biomarkers: the advantage and disadvantage. Clin Epigenetics. 2018;10:59

8. Lin XJ, Chong Y, Guo ZW, Xie C, Yang XJ, Zhang Q, Li SP, Xiong Y, Yuan Y, Min J, et al. A serum microRNA classifier for early detection of hepatocelIular carcinoma: a multicentre, retrospective, longitudinal biomarker identification study with a nested case-control study. Lancet Oncol. 2015;16(7):804-15.

9. Li L, Chen J, Chen X, Tang J, Guo H, Wang X, Qian J, Luo G, He F, Lu X, et al. Serum miRNAs as predictive and preventive biomarker for pre-clinical hepatocellular carcinoma. Cancer Lett. 2016;373(2):234-40.

10. Mozzoni P, Ampollini L, Goldoni M, Alinovi R, Tiseo M, Gnetti L, Carbognani P, Rusca M, Mutti A, Percesepe A, et al. MicroRNA expression in malignant pleural mesothelioma and asbestosis: a pilot study. Dis Markers. 2017:2017:9645940.

11. Cavalleri T, Angelici L, Favero C, Dioni L, Mensi C, Bareggi C, Palleschi A, Rimessi A, Consonni D, Bordini L, et al. Plasmatic extracellular vesicle microRNAs in malignant pleural mesothelioma and asbestos-exposed subjects suggest a 2-miRNA signature as potential biomarker of disease. PLOS ONE. 2017;12(5):e0176680.

12. Weber DG, Johnen G, Bryk O, Jockel KH, Bruning T. Identification of miRNA-103 in the cellular fraction of human peripheral blood as a potential biomarker for malignant mesothelioma - a pilot study. PLOS ONE. 2012;7(1):e30221.

13. Santarelli L, Strafella E, Staffolani S, Amati M, Emanuelli M, Sartini D, Pozzi V, Carbonari D, Bracci M, Pignotti E, et al. Association of MiR-126 with soluble mesothelin-related peptides, a marker for malignant mesothelioma. PLoS ONE. 2011:6(4):e18232.

14. Kirschner MB, Cheng YY, Badrian B, Kao SC, Creaney J, Edelman JJ, Armstrong NJ, Vallely MP, Musk AW, Robinson BW, et al. Increased circulating miR-625-3p: a potential biomarker for patients with malignant pleural mesothelioma. J Thorac Oncol. 2012;7(7):1184-91.

15. Weber DG, Gawrych K, Casjens S, Brik A, Lehnert M, Taeger D, Pesch B, Kollmeier J, Bauer TT, Johnen G, et al. Circulating miR-132-3p as a candidate diagnostic biomarker for malignant mesothelioma. Dis Markers. 2017;2017:9280170

16. Pesch B, Bruning T, Johnen G, Casjens S, Bonberg N, Taeger D, Muller A, Weber DG, Behrens T. Biomarker research with prospective study designs for the early detection of cancer. Biochem Biophys Acta. 2014;1844(5):874-83.

17. Johnen G, Burek K, Raiko I, Wichert K, Pesch B, Weber DG, Lehnert M, Casjens S, Hagemeyer O, Taeger D, et al. Prediagnostic detection of mesothelioma by circulating calretinin and mesothelin-a case-control comparison nested into a prospective cohort of asbestos-exposed workers. Sci Rep. 2018;8(1):14321.

18. Livak KJ, Schmittgen TD. Analysis of relative gene expression data using real-time quantitative PCR and the 2(-Delta Delta C(T)) Method. Methods. 2001;25(4):402-8

19. Lu J, Getz G, Miska EA, Alvarez-Saavedra E, Lamb J, Peck D, Sweet-Cordero A, Ebert BL, Mak RH, Ferrando AA, et al. MicroRNA expression profiles classify human cancers. Nature. 2005;435(7043):834-8.

20. Witwer KW. Circulating microRNA biomarker studies: pitfalls and potential solutions. Clin Chem. 2015;61(1):56-63.

21. Schwarzenbach H, Nishida N, Calin GA, Pantel K. Clinical relevance of circulating cell-free microRNAs in cancer. Nat Rev Clin Oncol. 2014;11(3):145-56. 
22. Blyuss O, Gentry-Maharaj A, Fourkala EO, Ryan A, Zaikin A, Menon U, Jacobs I, Timms JF. Serial patterns of ovarian cancer biomarkers in a prediagnosis longitudinal dataset. Biomed Res Int. 2015;2015:681416.

23. Johnen G, Gawrych K, Raiko I, Casjens S, Pesch B, Weber DG, Taeger D, Lehnert M, Kollmeier J, Bauer T, et al. Calretinin as a blood-based biomarker for mesothelioma. BMC Cancer. 2017;17(1):386.

24. Aguilar-Madrid G, Pesch B, Calderon-Aranda ES, Burek K, JimenezRamirez C, Juarez-Perez CA, Ochoa-Vazquez MD, Torre-Bouscoulet L, Acosta-Saavedra LC, Sada-Ovalle I, et al. Biomarkers for predicting malignant pleural mesothelioma in a mexican population. Int J Med Sci. 2018;15(9):883-91.

25. Weber DG, Casjens S, Johnen G, Bryk O, Raiko I, Pesch B, Kollmeier J, Bauer TT, Bruning T. Combination of MiR-103a-3p and mesothelin improves the biomarker performance of malignant mesothelioma diagnosis. PLoS ONE. 2014;9(12):e114483.

26. Kirschner MB, van Zandwijk N, Reid G. Cell-free microRNAs: potential biomarkers in need of standardized reporting. Front Genetics. 2013;4:56.

27. Pritchard CC, Kroh E, Wood B, Arroyo JD, Dougherty KJ, Miyaji MM, Tait JF, Tewari M. Blood cell origin of circulating microRNAs: a cautionary note for cancer biomarker studies. Cancer Prev Res. 2012;5(3):492-7.

28. Pizzamiglio S, Zanutto S, Ciniselli CM, Belfiore A, Bottelli S, Gariboldi M, Verderio P. A methodological procedure for evaluating the impact of hemolysis on circulating microRNAs. Oncol Lett. 2017;13(1):315-20.
Ready to submit your research? Choose BMC and benefit from:

- fast, convenient online submission

- thorough peer review by experienced researchers in your field

- rapid publication on acceptance

- support for research data, including large and complex data types

- gold Open Access which fosters wider collaboration and increased citations

- maximum visibility for your research: over $100 \mathrm{M}$ website views per year

At BMC, research is always in progress.

Learn more biomedcentral.com/submissions 